\title{
An appropriate way to extend the Banzhaf index for multiple levels of approval
}

November 13, 2020

\begin{abstract}
The Banzhaf power index for games admits several extensions if the players have more than two ordered voting options. In this paper we prove that the most intuitive and recognized extension of the index fails to preserve the desirability relation for games with more than three ordered input levels of approval, a failure that undermines the index to be a good measure of power.

This leads us to think of an alternative to the Banzhaf index for several input levels of approval. We propose a candidate for which it is proved that: 1 ) coincides with the Banzhaf index for simple games, 2) it is proportional to its known extension for three levels of approval, and 3) preserves the desirability relation regardless of the number of input levels of approval. This new index is based on measuring the total capacity the player has to alter the outcome. In addition, it can be expressed through a very appropriate mathematical formulation that greatly facilitates its computation.

Defining extensions of well-established notions in a wider context requires a careful analysis. Different extensions can provide complementary nuances and, when this occurs, none of them can be considered to be 'the' extension. As shown in this paper, this situation applies when trying to extend the Banzhaf power index from simple games to the broader context of games with several ordered input levels of approval.
\end{abstract}

Keywords Extensions of the Banzhaf index - Several ordered levels of input approval · Preservation of the desirability relation · Forms of criticality · Total criticality 


\section{Introduction}

The Banzhaf power index is possibly the most-recognized measure for evaluating the a priori power, as influence, of players in a simple game. The index was independently proposed by three authors: Penrose (1946), Banzhaf (1965) and Coleman (1971). A good survey on this index can be found in Dubey and Shapley (1979), and studies on limit approximations of the index with respect to weights can be found in Lindner and Machover (2004), Lindner and Owen (2007) and Kurz (2020). Several axiomatizations for the Banzhaf index in simple games or for the Banzhaf value in cooperative games (extension given in Owen (1975)) exist, see among others: Albizuri and Ruiz (2001); Alonso-Meijide et al. (2017); Barua et al. (2005); Casajus (2011, 2012); Dubey et al. (2005); Feltkamp (1995); Haller (2020); Lehrer (1988); Owen (1978). All of them have been very useful to better understand this power index and to highlight different features for it.

Felsenthal and Machover (1997, 1998) introduced the notion of 'ternary voting games' to model voting games that include abstention as an intermediate vote between 'yes' and 'no'. They provided an extension of the Banzhaf index to this wider context. In Tchantcho et al. (2008) the desirability (or influence) relation was introduced in this wider context, as a generalization of the desirability relation for simple games, Isbell (1958), and it was proved that the extension of the Banzhaf index given by Felsenthal and Machover preserves the desirability relation for ternary voting games. Limit approximations when abstention is allowed were studied in Lindner (2008) and some axiomatizations in Bernardi (2018) and Freixas and Lucchetti (2016).

In Freixas and Zwicker (2003) a more general model is proposed, that of $(j, k)$-simple games, a class of voting games in which voters may choose any of $j$ ordered levels of approval and $k$ stands for the number of possible aggregated ordered results. The United Nations Security Council and the US Congress (in each of the two Houses) are two real-life examples that can be treated as what we call $(3,2)$-simple games; there are three levels of approval in the input, and two levels in the output. See Freixas (2012) for the computation of the Banzhaf power index for the nations of the United Nations Security Council. $(j, 2)$-simple games are the voting structures analyzed in this paper, and briefly refer to them as $j$-simple games. The ternary voting games by Felsenthal and Machover correspond to 3-simple games and the Banzhaf index they defined naturally extends to $j$-simple games for $j>3$. In this paper we prove that this extension does not preserve the desirability relation for $j>3$ although it does for $j=3$, a failure that undermines it to be a good measure of power for $j$-simple games when $j>3$. For this reason we propose in this paper a reasonable alternative and prove that it preserves the desirability relation for all $j \geq 2$, so that it becomes a good candidate to be an appropriate extension of the Banzhaf power index in $j$-simple games for any $j \geq 3$. 
Extensions of other power notions, as the Johnston index (Johnston (1978)) or the Holler index (Holler (1982)) from simple games to $j$-simple games are considered in, for example, Kenfack et al. (2019); Courtin and Tchantcho (2020). As anticipated in Freixas (2012) a power index for simple games can admit more than one reasonable extension when passing from simple games to a broader context, in our case $j$-simple games. In a broader domain there are more nuances and considerations to make and, therefore, defining an extension of a well-established notion to a broader domain requires an adequate analysis on the likelihood of the proposed extension.

Classical cooperative games have given rise to several generalizations. Bolger (1986, 1990, 2002) deals with the so-called games with $n$ players and $r$ alternatives, in which the $r$ possible input alternatives are not ordered; each input alternative attracts its own coalition of supporting voters, and each such coalition is assigned an output cardinal value (so that the total output is an $r$-tuple of cardinal values). Amer et al. (1998) and Ono (2001) have continued Bolgers work, introducing the closely related $r$-games and multialternative games respectively.

The primary concern of all these authors is in developing value theory, in particular the Banzhaf value for the games they define. It should be noted that all the simple game examples considered by these authors do entail a linear ranking in the input and therefore fit into the context of $j$-simple games we consider in this paper.

Multi-choice games, Hsiao and Raghavan (1992, 1993), deserve special mention as an extension of cooperative games. In them players act at various levels of activity, which implies an ordered choice, and each partition is assigned an output cardinal value. The simple game version of multi-choice games is the class of $(j, 2)$-simple games, a particular class of $(j, k)$-simple games, Freixas and Zwicker (2003), in which the output admits several ordered options. If $k=2$ these options are either to pass the issue at hand or to reject it. In this paper we refer to $(j, 2)$-simple games as $j$-simple games. The $j$-simple game model we consider in this paper is distinguished from all the previous ones by the linear ordering of the input levels of approval.

In order to model possible uncertainties in a negotiaion process, other extensions of cooperative games have been considered. The notion of fuzzy game was first introduced by Aubin (1974) and since then different fuzzy approaches to cooperative games have been profusely developed in various directions by many scholars Borkotokey (2008); Meng et al. (2017); Jana and Roy (2019); Bhaumik et al. (2020). It is remarkable that a notion of the Banzhaf index in the context of uncertainty is provided in Gallego et al. (2014).

The rest of the paper is organized as follows. Section 2 introduces some preliminaries. Section 3 introduces the well-known extension of the (raw) Banzhaf index for $j$-simple games and another plausible alternative for it. Section 4 starts by providing some necessary technical results on raw indices that allow us to prove the main result, which establishes that the new extension, focused 
in what we call 'total criticality', preserves the desirability relation, while the well-known version does not. Section 5 provides simple expressions for the two indices that facilitate their computations. A conclusion ends the paper in Section 6

\section{Preliminaries}

We formally introduce $j$-simple games, the class of games we deal with in this paper. The notions are mainly taken from Freixas and Zwicker (2003).

Let $N$ be a finite set of voters. A $j$-partition of $N$ is a collection of $j$ mutually disjoint subsets of $N, S_{1}, \ldots, S_{j}$, such that $\cup_{k=1}^{j} S_{k}=N$. Note that any $S_{i}$ may be empty. A $j$-partition describes a division of players among $j$ levels of voting approval. We assume that these $j$ levels of approval are ordered and convey that level 1 corresponds to the highest level, while the last, level $j$, corresponds to the lowest one. Thus, voters in $S_{1}$ are those who vote for the highest level of approval, whereas those in $S_{j}$ vote for the lowest level of approval.

We denote by $J^{N}$ the set of all $j$-partitions on $N$. A partial order $\subseteq^{j}$ in the set $J^{N}$ is considered. If $S, T \in J^{N}$, then $S \subseteq{ }^{j} T$ means $S_{k} \subseteq \bigcup_{i=1}^{k} T_{i}$ for any $k=1, \ldots, j-1$. In words, $S$ is $j$-contained in $T$ if voters in $T$ are voting in the same or in a higher level than in $S$. We use $S \subset^{j} T$ if $S \subset^{j} T$ and $S \neq T$. The $j$-partitions $\mathcal{N}=(\emptyset, \ldots, \emptyset, N)$ and $\mathcal{M}=(N, \emptyset, \ldots, \emptyset)$ are respectively the minimum and maximum for the order $\subseteq^{j}$.

In the next definition we consider a voting situation in which voters can vote among several ordered alternatives and the collective or aggregated decision is binary and represented by the numbers 0 and 1 , where 0 means that the submitted proposal is defeated and 1 that it is passed.

Definition 1 Let $N$ be a finite set and $J^{N}$ be the set of all $j$-partitions on $N$. A $j$-simple game on $N$ is a function $v: J^{N} \rightarrow\{0,1\}$ such that: $\left.i\right)$ it is monotonic: if $S \subset^{j} T$ then $v(S) \leq v(T)$; ii) $v(\mathcal{N})=0$ and $v(\mathcal{M})=1$.

We denote by $\mathcal{S}_{N}$ the set of all $j$-simple games on the finite set $N$ for any value of $j \geq 2$. Note that simple games are 2 -simple games since for any bipartition $S=\left(S_{1}, S_{2}\right)$ the first component $S_{1}$ is identified with the set of 'yes'-voters and $S_{2}=N \backslash S_{1}$ with the set of 'no'-voters. Thus, any bipartition is in one-to-one correspondence with coalition $S_{1}$. Note also that 3-simple games can be interpreted as ternary voting games, as considered by Felsenthal and Machover (1997), if the first level of approval correspond to voting 'yes', the second level to abstain and the third level to voting 'no'.

A $j$-partition $S$ is winning if $v(S)=1$. The set of winning $j$-partitions of $v$ is denoted by $W(v)=\left\{S \in J^{N}: v(S)=1\right\}$ and the set of minimal winning $j$-partitions by $W^{m}(v)=\left\{S \in W(v): T \notin W(v)\right.$ if $\left.T \subset^{j} S\right\}$. 
Let us introduce the following notation. From a given $j$-partition $S=$ $\left(S_{1}, \ldots, S_{j}\right)$ such that $a \in S_{i}$ for some $i<j$, we define the $j$-partition $S_{a_{i \rightarrow k}}$ in which player $a$ has moved from the level of approval $i$ to the inferior level $k(i<k \leq j)$.

$$
S_{a_{i \rightarrow k}}=\left(S_{1}, \ldots, S_{i-1}, S_{i} \backslash\{a\}, S_{i+1}, \ldots, S_{k-1}, S_{k} \cup\{a\}, S_{k+1}, \ldots, S_{j}\right) .
$$

The idea we pursue with this notation is to define the $(i, k)$-marginal contribution of a player $a$ for a given $j$-partition $S$ and game $v$ :

$$
m_{k}^{i}(v, S, a)=v(S)-v\left(S_{a_{i \rightarrow k}}\right) \quad \text { if } a \in S_{i} .
$$

When we focus on two voters and a $j$-partition we use the notation $\left(S, a_{i}, b_{k}\right)$ which denotes a $j$-partition $S$ emphasizing that $a \in S_{i}$ and $b \in S_{k}$ with $i<k$. $\left(S, b_{i}, a_{k}\right)$ is obtained from $\left(S, a_{i}, b_{k}\right)$ by only swapping the input levels of $a$ and $b$.

The desirability relation introduced for simple games $(j=2)$ in Isbell (1958) and for voting games with abstention $(j=3)$ in Tchantcho et al. (2008), can be extended to more than 3 levels of approval, and we do it in Definition 2. For studies on the desirability relation for simple games we refer to Einy (1985) and Taylor and Zwicker (1999), and to Freixas et al. (2014a b) for games with three ordered levels of approval.

Definition 2 (The desirability relation) Let $v$ be a $j$-simple game on $N$ for any $j \geq 2$ and let $a, b \in N$ and $1 \leq i<k \leq j$.

- $a$ is more $(i, k)$-desirable than $b$ (denoted by $\left.a \succsim_{k}^{i} b\right)$ if: $v\left(S, a_{i}, b_{k}\right) \geq v\left(S, b_{i}, a_{k}\right)$ for all $j$-partition $\left(S, a_{i}, b_{k}\right)$.

- $a$ is strictly more $(i, k)$-desirable than $b$ (denoted by $\left.a \succ_{k}^{i} b\right)$ if: $a \succsim_{k}^{i} b$ and $v\left(S, a_{i}, b_{k}\right)>v\left(S, b_{i}, a_{k}\right)$ for some $j$-partition $\left(S, a_{i}, b_{k}\right)$.

- $a$ is equally $(i, k)$-desirable than $b$ (denoted by $a \approx_{k}^{i} b$ ) if: $a \succsim_{k}^{i} b$ and $b \succsim_{k}^{i} a$

- $a$ is more desirable than $b$ (denoted by $a \succsim b)$ if: $a \succsim_{k}^{i} b$ for every pair $i$ and $k$ with $1 \leq i<k \leq j$.

- $a$ is strictly more desirable than $b$ (denoted by $a \succ b$ ) if: $a \succsim b$ and $a \succ_{k}^{i} b$ for some $i, k$ with $1 \leq i<k \leq j$.

- $a$ is equally desirable than $b$ (denoted by $a \approx b$ ) if: $a \succsim b$ and $b \succsim a$.

Tchantcho et al. (2008) proved, for $j=3$, that the desirability relation is reflexive but not necessarily transitive. Properties that trivially extend to $j>3$.

\section{A new extension of the raw Banzhaf power index}

As occurs in simple games, any extension of the Banzhaf index for $j$-simple games admits two versions: the absolute and the relative. They are proportional and just differ on how the raw Banzhaf index is normalized: the absolute 
version is divided by the number of alternatives $j$ to the power of $n-1$ where $n$ is the number of players of the game, while the relative Banzhaf index is rescaled so that the sum of the index for all players is 1 . As the absolute and relative versions rank players equally, from now on we will only consider raw indices.

We consider two plausible extensions of the raw Banzhaf index for $j$-simple games with $j \geq 2$. Of course, they coincide with the raw Banzhaf index for simple games when $j=2$. The first extension (in Subsection 3.1) has been widely used, however the second one (in Subsection 3.2 is a novel proposal.

\subsection{The 1-critical raw Banzhaf index}

Let $a \in N, v$ be a $j$-simple game on $N, S$ be a $j$-partition with $a \in S_{i}$ and $i<j$. Let

$$
\eta_{a}^{1}(v, i, S)=m_{i+1}^{i}(v, S, a) .
$$

Definition 3 (The 1-critical raw Banzhaf index) For any $v \in \mathcal{S}_{N}$ and any voter $a \in N$, the 1-critical raw Banzhaf index is defined as

$$
\eta_{a}^{1}(v)=\sum_{i=1}^{j-1} \sum_{\substack{S \in J^{N} \\ a \in S_{i}}} \eta_{a}^{1}(v, i, S) .
$$

This raw index intends to capture the sensitivity the player has to change the outcome by changing her vote to the immediate consecutive inferior level of approval.

This index is considered for $j=3$ in Felsenthal and Machover (1997), and studied among others in Parker (2012); Pongou et al. (2011); Tchantcho et al. (2008); Kenfack et al. (2019). It has also been considered for $j \geq 3$ in Freixas (2005). Note that Equation (1) can also be written as

$$
\eta_{a}^{1}(v)=\sum_{\substack{S \in J^{N} \\ a \in S_{1}}} m_{j}^{1}(v, S, a),
$$

which allows a shorter computation of the index.

\subsection{The totally critical raw Banzhaf index}

Instead of focusing on the sensitivity the player has in the game, we may consider the total capacity the player has to influence the outcome. This is captured by considering all types of marginal contributions, not only those 
affecting consecutive levels of approval. We denote this raw index by $\eta^{t}(v)$ and refer to it as the totally critical raw index (or, briefly, $t$-critical raw index). As far as we know, this is a new index. In this paper we prove that it plays a relevant role in contrast with the well-known 1-critical raw Banzhaf index.

Let $a \in N, v$ be a $j$-simple game on $N, S$ be a $j$-partition with $a \in S_{i}$ and $i<j$. Consider

$$
\eta_{a}^{t}(v, i, S)=\sum_{k=i+1}^{j} m_{k}^{i}(v, S, a)
$$

Observe that

$$
\eta_{a}^{t}(v, i, S)=\sum_{k=i+1}^{j} m_{k}^{i}(v, S, a)=(j-i) v(S)-\sum_{k=i+1}^{j} v\left(S_{a_{i \rightarrow k}}\right) .
$$

Definition 4 (The totally critical raw Banzhaf index) For any $v \in \mathcal{S}_{N}$ and any voter $a \in N$, the $t$-critical raw Banzhaf index is defined as

$$
\eta_{a}^{t}(v)=\sum_{i=1}^{j-1} \sum_{\substack{S \in J^{N} \\ a \in S_{i}}} \eta_{a}^{t}(v, i, S)
$$

Example 1 The main shareholder of a company $(a)$ and its manager $(b)$ must decide whether or not to undertake a major project. Both separately assess the pros and cons of it and each one of them expresses the degree of support to the project: from 1 (maximum support) to $j$ (minimum support), for a prefixed $j \geq 4$. The decision is made according to the $j$-simple game $v$ on $N=\{a, b\}$ defined as

$$
W^{m}(v)=\{(a, \emptyset, \emptyset, b, \underbrace{\emptyset, \ldots, \emptyset}_{j-4}),(b, \emptyset, \emptyset, a, \underbrace{\emptyset, \ldots, \emptyset}_{j-4}),(\emptyset, a, b, \emptyset, \underbrace{\emptyset, \ldots, \emptyset}_{j-4})\} .
$$

We are concerned with the following questions:

- What is the relative importance of each of them in the decision making as described by $v$ ?

- If, with their decision, they were rewarded with an amount to be distributed, what would be a fair deal between them?

According to Felsenthal and Machover (1998) the first question can be solved by dealing with power as influence and the second with power as a payoff. An ideal measure to solve the problem would be the one that best generalizes the Banzhaf's power index.

In the game $v$ it holds that $a \succ b$ because $a \succsim_{k}^{i} b$ for all $1 \leq i<k \leq j$ and $a \succ_{3}^{2} b$ since

$$
(\emptyset, a, b, \emptyset, \underbrace{\emptyset, \ldots, \emptyset}_{j-4}) \in W \text { and }(\emptyset, b, a, \emptyset, \underbrace{\emptyset, \ldots, \emptyset}_{j-4}) \notin W .
$$


Table 1 shows the game for $j=4$ and $j=5$. If it was $j>5$ we would just need to add right columns and below rows with zeros.

\begin{tabular}{|c|c|c|c|c|}
\hline$\downarrow a \mid \stackrel{b}{\rightarrow}$ & 1 & 2 & 3 & 4 \\
\hline \hline 1 & 1 & 1 & 1 & 1 \\
2 & 1 & 1 & 1 & 0 \\
3 & 1 & 0 & 0 & 0 \\
4 & 1 & 0 & 0 & 0 \\
\hline
\end{tabular}

\begin{tabular}{|c|c|c|c|c|c|}
\hline$\downarrow a \mid \stackrel{b}{\rightarrow}$ & 1 & 2 & 3 & 4 & 5 \\
\hline \hline 1 & 1 & 1 & 1 & 1 & 0 \\
2 & 1 & 1 & 1 & 0 & 0 \\
3 & 1 & 0 & 0 & 0 & 0 \\
4 & 1 & 0 & 0 & 0 & 0 \\
5 & 0 & 0 & 0 & 0 & 0 \\
\hline
\end{tabular}

Table 1 Each label in the first columns indicate the level of approval chosen by $a$ and each label in the first rows indicate the level of approval chosen by $b$. Every combination gives a winning $j$-partition (1) or a losing $j$-partition (0) for $j=4$ and $j=5$ respectively.

The raw index $\eta_{a}^{1}(v)$ is obtained by just adding the differences between the numbers in the first row with those in the last one. Instead, $\eta_{b}^{1}(v)$ is obtained analogously by replacing rows by columns. For $j=4$ we have $\eta_{a}^{1}(v)=$ $\eta_{b}^{1}(v)=(1-1)+3(1-0)=3$ and for $j>4$ we have $\eta_{a}^{1}(v)=\eta_{b}^{1}(v)=$ $4(1-0)+(j-4)(0-0)=4$.

The raw index $\eta_{a}^{t}(v)$ is obtained by adding for all columns, all the substractions of numbers occupying a position above the other. For instance, when computing $\eta_{a}^{t}(v)$ for $j=5$ we obtain for the first column: $(3(1-1)+$ $1(1-0))+(2(1-1)+1(1-0))+(1(1-1)+1(1-0)+(1-0))=4$. Analogous computations for the other columns are: $(1(1-1)+3(1-0))+(3(1-0))=6$ for the second, $(1(1-1)+3(1-0))+(3(1-0))=6$ for the third, $(4(1-0))=4$, for the fourth and 0 for the fifth. So that $\eta_{a}^{t}(v)=20$.

Analogously, when computing $\eta_{b}^{t}(v)$, we obtain $4,6,4,4$, and 0 for the respective rows, so that $\eta_{b}^{t}(v)=18$.

Note that the values of $\eta_{a}^{1}(v)$ and $\eta_{b}^{1}(v)$ are equal to 4 for all $j>4$, while the values of $\eta_{a}^{t}(v)$ and $\eta_{b}^{t}(v)$ start at 11 and 9 (values for $j=4$ ) respectively and are increased by nine for each unit that $j$ exceeds 4 .

$$
\begin{gathered}
\left(\eta_{a}^{1}(v), \eta_{b}^{1}(v)\right)= \begin{cases}(3,3) \text { if } j=4, \\
(4,4) \text { if } j>4,\end{cases} \\
\left(\eta_{a}^{t}(v), \eta_{b}^{t}(v)\right)=(11+9(j-4), 9+9(j-4)) .
\end{gathered}
$$

Thus, $\eta_{a}^{1}(v)=\eta_{b}^{1}(v)$, while $\eta_{a}^{t}(v)>\eta_{b}^{t}(v)$.

Thus, voter $a$ strictly dominates $b$ by the desirability relation, but $\eta^{1}$, the standard extension of the Banzhaf index, does not reflect the better position of $a$ over $b$. Instead, $\eta^{t}$ does. This example is not an isolated case: as shown in Section 4 the raw index $\eta^{t}$ preserves the desirability relation. 


\section{The main result: preservation of the desirability relation}

A good criterion for deciding whether a (raw) power index accurately reflects the strength relations of voters in the game is the preservation of the desirability relation. Thus, an arbitrary power index $P$ is a good yardstick for measuring power if it fulfills:

$$
\begin{aligned}
& a \approx b \Rightarrow P_{a}=P_{b}, \\
& a \succ b \Rightarrow P_{a}>P_{b} .
\end{aligned}
$$

a weaker version is

$$
\begin{aligned}
& a \approx b \Rightarrow P_{a}=P_{b}, \\
& a \succ b \Rightarrow P_{a} \geq P_{b} .
\end{aligned}
$$

In that case we say that the (raw) power index is not contradictory with the desirability relation.

Both, the Banzhaf index for simple games and the usual extension of the Banzhaf index for ternary voting games preserve the desirability, i.e., the 1critical raw Banzhaf index preserves the desirability relation for the particular cases $j=2$ and $j=3$. In this paper we deal with this issue for $j \geq 4$ and also for the $t$-critical raw Banzhaf index for all $j \geq 3$.

Before stating the main result we need the following two technical lemmas that will be used to compare the raw indices of two players.

Lemma 1 Let $v$ be a $j$-simple game on $N$ with $j \geq 2$ and $\{a, b\} \subseteq N$. Then,

$$
\eta_{a}^{1}(v)-\eta_{b}^{1}(v)=\sum_{k=2}^{j} \sum_{i=1}^{k-1} \sum_{\substack{S \in J^{N} \\ a \in S_{i} \\ b \in S_{k}}} \gamma^{1}(i, k) \cdot\left[v\left(S, a_{i}, b_{k}\right)-v\left(S, b_{i}, a_{k}\right)\right]
$$

where

$$
\gamma^{1}(i, k)= \begin{cases}2, & \text { if } i=1, k=j \\ 0, & \text { if } 1<i<k<j \\ 1, & \text { otherwise }\end{cases}
$$

Proof The expression for the difference $\eta_{a}^{1}(v)-\eta_{b}^{1}(v)$ in Equation 6 follows from the expression of $\eta_{a}^{1}(v)$ in (1), which after simplification becomes

$$
\eta_{a}^{1}(v)=\sum_{\substack{S \in J^{N} \\ a \in S_{1}}} v(S)-\sum_{\substack{S \in J^{N} \\ a \in S_{j}}} v(S)
$$

Then, Equation $(6)$ is derived from the following:

- Assume $S$ is a $j$-partition such that $\{a, b\} \subseteq S_{1}$. Then, $v(S)$ is cancelled when computing $\eta_{a}^{1}(v)-\eta_{b}^{1}(v)$ because it appears once in the first addend of the right-hand side expression in (7) for both $\eta_{a}^{1}(v)$ and $\eta_{b}^{1}(v)$. 
- Assume $S$ is a $j$-partition such that $\{a, b\} \subseteq S_{j}$. Then, $v(S)$ is cancelled when computing $\eta_{a}^{1}(v)-\eta_{b}^{1}(v)$ because it appears once in the second addend of the right-hand side expression in (7) for both $\eta_{a}^{1}(v)$ and $\eta_{b}^{1}(v)$.

- Assume $S$ is a $j$-partition such that $\{a, b\} \subseteq S_{i}$ with $1<i<j$. Then, $v(S)$ does not appear neither in $\eta_{a}^{1}(v)$ nor in $\eta_{b}^{1}(v)$. Thus, it does not appear in $\eta_{a}^{1}(v)-\eta_{b}^{1}(v)$

From these three items it is clear that only $j$-partitions where $a$ and $b$ occupy different positions may appear in the explicit expression of $\eta_{a}^{1}(v)-$ $\eta_{b}^{1}(v)$. The next four items provide the coefficients in Equation (6).

- Assume $S$ is a $j$-partition such that $a \in S_{1}$ and $b \in S_{k}$ with $1<k<j$. Then, $v\left(S, a_{1}, b_{k}\right)$ appears with coefficient 1 in $\eta_{a}^{1}(v)$ and does not appear in $\eta_{b}^{1}(v)$, while $v\left(S, b_{1}, a_{k}\right)$ appears with coefficient 1 in $\eta_{b}^{1}(v)$ and does not appear in $\eta_{a}^{1}(v)$. Thus, $v\left(S, a_{1}, b_{k}\right)-v\left(S, b_{1}, a_{k}\right)$ appears in $\eta_{a}^{1}(v)-\eta_{b}^{1}(v)$ with 1 as a multiplicative coefficient. Hence, $\gamma^{1}(1, k)=1$ for $1<k<j$.

- Assume $S$ is a $j$-partition such that $a \in S_{1}$ and $b \in S_{j}$. Then, $v\left(S, a_{1}, b_{j}\right)$ appears with coefficient 1 in $\eta_{a}^{1}(v)$ and appears with coefficient -1 in $\eta_{b}^{1}(v)$, while $v\left(S, b_{1}, a_{j}\right)$ appears with coefficient -1 in $\eta_{a}^{1}(v)$ and appears with coefficient 1 in $\eta_{b}^{1}(v)$. Thus, $v\left(S, a_{1}, b_{j}\right)-v\left(S, b_{1}, a_{j}\right)$ appears in $\eta_{a}^{1}(v)-\eta_{b}^{1}(v)$ with 2 as a multiplicative coefficient. Hence, $\gamma^{1}(1, j)=2$.

- Assume $S$ is a $j$-partition such that $a \in S_{k}$ with $1<k<j$ and $b \in S_{j}$. Then, $v\left(S, a_{k}, b_{j}\right)$ does not appear in $\eta_{a}^{1}(v)$ and appears with coefficient -1 in $\eta_{b}^{1}(v)$, while $v\left(S, b_{k}, a_{j}\right)$ appears with coefficient -1 in $\eta_{a}^{1}(v)$ and does not appear in $\eta_{b}^{1}(v)$. Thus, $v\left(S, a_{k}, b_{j}\right)-v\left(S, b_{k}, a_{j}\right)$ appears in $\eta_{a}^{1}(v)-\eta_{b}^{1}(v)$ with 1 as a multiplicative coefficient. Hence, $\gamma^{1}(k, j)=1$ for $1<k<j$.

- Assume $S$ is a $j$-partition such that $a \in S_{i}$ with $1<i<j$ and $b \in S_{k}$ with $1<i<k<j$. Then, $v\left(S, a_{i}, b_{k}\right)$ neither appears in $\eta_{a}^{1}(v)$ nor in $\eta_{b}^{1}(v)$, and the same occurs for $v\left(S, b_{i}, a_{k}\right)$. Thus, $v\left(S, a_{i}, b_{k}\right)-v\left(S, b_{i}, a_{k}\right)$ does not appear in $\eta_{a}^{1}(v)-\eta_{b}^{1}(v)$. Hence, $\gamma^{1}(i, k)=0$ for $1<i<k<j$.

Lemma 2 Let $v$ be a $j$-simple game on $N$ with $j \geq 2$ and $\{a, b\} \subseteq N$. Then,

$$
\eta_{a}^{t}(v)-\eta_{b}^{t}(v)=\sum_{k=2}^{j} \sum_{i=1}^{k-1} \sum_{\substack{S \in J^{N} \\ a \in S_{i} \\ b \in S_{k}}} \gamma^{t}(i, k) \cdot\left[v\left(S, a_{i}, b_{k}\right)-v\left(S, b_{i}, a_{k}\right)\right]
$$

where

$$
\gamma^{t}(i, k)=2(k-i)
$$

Proof The expression for the difference $\eta_{a}^{t}(v)-\eta_{b}^{t}(v)$ in Equation (8) follows from the expression of $\eta_{a}^{t}(v)$ in Equation (3). By using (2) we have:

$$
\eta_{a}^{t}(v)=\sum_{i=1}^{j}(j-2 i+1) \sum_{\substack{S \in J^{N} \\ a \in S_{i}}} v(S) .
$$


Observe now that $v\left(S, a_{i}, b_{k}\right)$ has $(j-2 i+1)$ as a multiplicative coefficient in $\eta_{a}^{t}(v)$, see the right-hand expression in $(9)$, and has $(j-2 k+1)$ as a multiplicative coefficient in $\eta_{b}^{t}(v)$.

On the other hand, $v\left(S, b_{i}, a_{k}\right)$ has $(j-2 k+1)$ as a multiplicative coefficient in $\eta_{a}^{t}(v)$ and has $(j-2 i+1)$ as a multiplicative coefficient in $\eta_{b}^{t}(v)$. Hence, the coefficient of $v\left(S, a_{i}, b_{k}\right)-v\left(S, b_{i}, a_{k}\right)$ in the expression of $\eta_{a}^{t}(v)-\eta_{b}^{t}(v)$ is $\gamma^{t}(i, k)=(j-2 i+1)-(j-2 k+1)=2(k-i)$.

Note that for $j=2$ the raw indices $\eta_{a}^{1}(v)$ and $\eta_{a}^{t}(v)$ coincide, and consequently the differences $\eta_{a}^{1}(v)-\eta_{b}^{1}(v)$ and $\eta_{a}^{t}(v)-\eta_{b}^{t}(v)$ also coincide. Instead, for $j>2$ all these expressions are different.

The following theorem establishes that the (raw) total critical index preserves the desirability relation and therefore has the potential to be a good yardstick to measure power in the same spirit as the Banzhaf index does for simple games. Note that the step from $j=3$ to $j=4$ becomes crucial.

Theorem 1 Let $\mathcal{S}_{N}$ be the class of all $j$-simple games on $N$ for all $j \geq 2$, and $\mathcal{S}_{N}(j)$ be the class of all $j$-simple games for some $j \geq 2$.

a. $\eta^{t}$ respects the desirability relation for all $v \in \mathcal{S}_{N}$.

b. $\eta^{1}$ is not contradictory with the desirability relation for all $v \in \mathcal{S}_{N}$.

c. $\eta^{1}$ respects the desirability relation for all $v \in \mathcal{S}_{N}(j)$ if and only if $j \leq 3$.

Proof a. $\eta^{t}$ respects the desirability relation for all $v \in \mathcal{S}_{N}$.

- $a \approx b$ implies $v\left(S, a_{i}, b_{k}\right)=v\left(S, b_{i}, a_{k}\right)$ for every $\left(S, a_{i}, b_{k}\right)$ and for all $i, k$ with $1 \leq i<k \leq j$. This implication together with Lemma2 2 implies $\eta_{a}^{t}(v)=\eta_{b}^{t}(v)$.

- $a \succsim b$ implies $v\left(S, a_{i}, b_{k}\right) \geq v\left(S, b_{i}, a_{k}\right)$ for every $\left(S, a_{i}, b_{k}\right)$ and for all $i, k$ with $1 \leq i<k \leq j$. Moreover, $\gamma^{t}(i, k)>0$ for all pair $(i, k)$ with $1 \leq i<k \leq j$. Thus, we deduce $\eta_{a}^{t}(v) \geq \eta_{b}^{t}(v)$ from Lemma 2

- $a \succ b$ implies $v\left(S, a_{i}, b_{k}\right) \geq v\left(S, b_{i}, a_{k}\right)$ for every $\left(S, a_{i}, b_{k}\right)$ and for all $i, k$ with $1 \leq i<k \leq j$, and $v\left(T, a_{i}, b_{k}\right)>v\left(T, b_{i}, a_{k}\right)$ for some $\left(T, a_{i}, b_{k}\right)$ and some $i, k$ with $1 \leq i<k \leq j$. As $\gamma^{t}(i, k)>0$ for all $i, k$ with $1 \leq i<k \leq j$ we deduce from Lemma 2 that $\eta_{a}^{t}(v)>\eta_{b}^{t}(v)$.

b. $\eta^{1}$ is not contradictory with the desirability relation for all $v \in \mathcal{S}_{N}$.

It follows the same guidelines of the two first items above for $\eta^{t}$ because we may use Lemma 1 and that $\gamma^{1}(i, k) \geq 0$ for all pair $(i, k)$ with $1 \leq i<k \leq j$ to deduce that: $a \approx b$ implies $\eta_{a}^{1}(v)=\eta_{b}^{1}(v)$, and $a \succsim b$ implies $\eta_{a}^{1}(v) \geq$ $\eta_{b}^{1}(v) \cdot \gamma^{1}(i, k) \geq 0$ only guarantees that $a \succ b$ implies $\eta_{a}^{1}(v) \geq \eta_{b}^{1}(v)$, but the inequality is not necessarily strict.

c. $\eta^{1}$ respects the desirability relation for all $v \in \mathcal{S}_{N}(j)$ if and only if $j \leq 3$.

- $(\Leftarrow) a \succ b$ implies $\eta_{a}^{1}(v)-\eta_{b}^{1}(v)>0$ for $j=2$ or $j=3$ since $\gamma^{1}(i, k)>0$ for all pair $(i, k)$ with $1 \leq i<k \leq j$. In fact if, $j=2$ then $\gamma^{1}(1,2)=$ $2>0$ and if $j=3$, then $\gamma^{1}(1,2)=1>0, \gamma^{1}(1,3)=2>0$ and $\gamma^{1}(2,3)=1>0$. 
- $(\Rightarrow)$ If $j \geq 4$ consider the game in Example 11. It holds $a \succ b$ but $\eta_{a}^{1}(v)=\eta_{b}^{1}(v)$.

\section{Simple explicit expressions for the two power indices}

The purpose of this section is to express $\eta^{1}$ and $\eta^{t}$ in a more convenient and simple way. Let $v$ be a $j$-simple game on $N, a \in N$ and set

$$
v_{a_{i}}=\sum_{\substack{S \in J^{N} \\ a \in S_{i}}} v(S) \quad \text { for all } i=1, \ldots, j .
$$

Then, both $\eta_{a}^{1}(v)$ and $\eta_{a}^{t}(v)$ can be written as

$$
\sum_{i=1}^{j} c_{i} \cdot v_{a_{i}}
$$

for some integer coefficients $c_{i}$ verifying the following properties:

a. $\sum_{i=1}^{j} c_{i}=0$,

b. $c_{i} \geq c_{i+1}$ for all $i=1, \ldots, j-1$, and

c. $c_{i}=-c_{j-i+1}$ for all $i=1, \ldots\left\lceil\frac{j}{2}\right\rceil$.

In particular, Equation (7) can be written as

$$
\eta_{a}^{1}(v)=v_{a_{1}}-v_{a_{j}}
$$

i.e., the coefficients $c_{i}$ for $\eta_{a}^{1}(v)$ are:

$$
c_{i}=\left\{\begin{aligned}
1, & \text { if } i=1, \\
-1, & \text { if } i=j \\
0, & \text { otherwise }
\end{aligned}\right.
$$

and Equation $(9)$ can be written as

$$
\eta_{a}^{t}(v)=\sum_{i=1}^{j}(j-2 i+1) v_{a_{i}}
$$

i.e., the coefficients $c_{i}$ for $\eta_{a}^{t}(v)$ are: $c_{i}=j-2 i+1$, which decrease two by two. The strict decrease of the coefficients $c_{i}\left(c_{i}>c_{i+1}\right.$ for all $\left.i=1, \ldots, j-1\right)$ for $\eta^{t}$ plays a decisive role for the preservation of the desirability relation in next subsection. Note also the coincidence of the coefficients for $\eta_{a}^{1}(v)$ and $\eta_{a}^{t}(v)$ if $j=2$ and the similarity for $j=3$, that is, the coefficients of $\eta_{a}^{t}(v)$ in 13 . double those of $\eta_{a}^{1}(v)$ in 12 , so that $\eta^{t}=2 \eta^{1}$. But, for $j \geq 4$ the distinction of the two indices becomes drastic and, therefore, it is very important to know what we intend to measure. 
It is clear then that the two indices coincide for $j=2$ and for $j=3$ they only differ by a proportionality factor equal to 2 . It is only from $j>3$ when the divergence between both measures is manifested, which forces us to rethink which measure is best suited to extend the Banzhaf measure for $j=4$ or more ordered input alternatives. The result in the next subsection positions $\eta^{t}$ more favorably.

If, additionally to the three conditions listed above to the integer coefficients $c_{i}$, we demand strict and linear decrease, i.e.,
a. $\sum_{i=1}^{j} c_{i}=0$,
b. $c_{i}>c_{i+1}$ for all $i=1, \ldots, j-1$,
c. $c_{i}=-c_{j-i+1}$ for all $i=1, \ldots\left\lceil\frac{j}{2}\right\rceil$, and
d. $c_{i}-c_{i+1}=k$ for $i=1, \ldots, j-1$ and some $k>0$.

Then $k$ needs to be an even positive integer and the minimum choice $k=2$ leads to the raw index $\eta^{t}$, while the remaining values for $k$ lead to the affine index $(k / 2) \eta^{t}$.

All indices defined in (11) can be easily computed. For Example 1 it is immediate to compute the numbers in 100 :

$$
\begin{aligned}
& v_{a_{1}}=4, \quad v_{a_{2}}=3, \quad v_{a_{3}}=1, \quad v_{a_{4}}=1, \quad v_{a_{k}}=0 \text { for all } 4<k \leq j \\
& v_{b_{1}}=4, \quad v_{b_{2}}=2, \quad v_{b_{3}}=2, \quad v_{b_{4}}=1, \quad v_{a_{k}}=0 \text { for all } 4<k \leq j
\end{aligned}
$$

Note that these numbers for $a$ are respectively the sums of the numbers in the rows in Table 1 and the numbers for $b$ are the sums of the numbers in the columns in Table 1. Thus, Equation $(12)$ and Equation 13 lead us to the obtained result for $\eta^{1}$ in (4) and for $\eta^{t}$ in (5). If we consider the case $j=4$, the distribution of power in proportion 11:9 for $a$ and $b$ is more adequate and fair than an equal distribution. Similarly, if $j=5$, the distribution $20: 18$ is preferable than the equal distribution. Note, however, that when $j$ tends to infinity the proportion $\eta_{a}^{t}: \eta_{b}^{t}$ tends to 1 .

\section{Conclusion}

The paper illustrates that defining extensions of power indices from simple games to broader contexts is not a trivial matter. We consider first 'the' standard extension of the Banzhaf index for simple games to $j$-simple games, i.e., games with several ordered levels of approval for voters with a binary collective decision. This extension has been defined almost unanimously by several authors and is the one that results from considering the raw index $\eta^{1}$. Tchantcho et al. (2008) already proved that $\eta^{1}$ respects the desirability relation for $j=3$. However, we show in this paper that $\eta^{1}$ fails to preserve it for $j>3$, property that in our opinion disqualifies it to be considered a good measure of power. 
When passing from simple games to $j$-simple games for $j \geq 3$, one should not only take into account the minimal marginal contributions, but all of them. From this observation it results a new (raw) index, $\eta^{t}$, that preserves the desirability relation regardless of the number of input levels, $j$, considered.

The raw index $\eta^{t}$ considered in this paper is a new alternative version to the raw index $\eta^{1}$. The two indices considered measure different aspects. $\eta^{1}$ is a sensitivity index, valid to measure the player's ability to alter the output through minimal changes in her vote. $\eta^{t}$ measures the total player's ability to alter the output by means of arbitrary changes in her vote, not necessarily being minimal changes.

The generalization of a power index from simple games to broader contexts may admit, as reflected in this paper, several interpretations, that provide different complementary nuances. Therefore we think that it is not appropriate to define 'the' extension of a certain value but rather 'an' or 'some' extensions of it. The result in this paper provides arguments in favor of $\eta^{t}$ to be an extension of the Banzhaf power index for an arbitrary number of input levels of approval.

Two respective axiomatizations for $\eta^{1}$ were provided in Freixas (2020) in the contexts of $j$-simple games and $j$-cooperative games. Analogously, the raw index $\eta^{t}$, as formulated in Definition 3.2 is obviously well-defined for $j$-cooperative games. It would be an interesting problem for future research to provide axiomatizations for this new index for $j$-simple games and for $j$ cooperative games.

We highlighting the simplicity of the explicit formulas for $\eta_{a}^{1}(v)$ in Equation (12) and for $\eta_{a}^{t}(v)$ in Equation (13), which have the potential to be very useful in the $j$-cooperative setting.

The analysis done in this paper, focused on the Banzhaf index, can be extended to other power indices. Thus, we encourage similar studies concerning the adequacy of extending power indices or values when more than two ordered levels of approval are allowed to voters.

\section{Acknowledgements}

We are grateful to the referees of this work whose comments have contributed to improve the final presentation.

\section{References}

Albizuri, M.J. and Ruiz, L.M. (2001) A new axiomatization of the Banzhaf semivalue. Spanish Economic Review, 3: 97-109.

Alonso-Meijide, J.M., Álvarez-Mozos, M. and Fiestras-Janeiro, M.G. (2017) Power indices and minimal winning coalitions for simple games in partition function form. Group Decision and Negotiation, 26:1231-1245. 
Amer, R., Carreras, F. and Magaña, A. (1998) The Banzhaf-Coleman index for games with r alternatives. Optimization, 44:175-198.

Aubin, J.P. (1974). Coeur et valeur des jeux flous à paiements latéraux. $C R$ Hebdomad D 279-A:891-894.

Banzhaf, J.F. (1965). Weighted voting doesn't work: a mathematical analysis. Rutgers Law Review 19:317-343.

Barua, R., Chakravarty, S.R., and Roy, S. (2005) A new characterization of the Banzhaf index of power. International Game Theory Review, 7: 545-553.

Bernardi, G. (2018) A new axiomatization of the Banzhaf index for games with abstention. Group Decision and Negotiation, 27: 165-177.

Bhaumik, A., Roy, S.K., and Weber, G.W. (2020) Hesitant interval-valued intuitionistic fuzzy-liguistic term set approach in prisoners dilemma game theory using TOPSIS: a case study on Human-trafficking. Central European Journal of Operations Research, 28: $797-816$.

Bolger, E. (1986). Power indices for multicandidate voting games. Int J Game Theory 15:175-186.

Bolger, E. (1990). A characterization of an extension of the Banzhaf value for multicandidate voting games. SIAM J Discret Math 3:466-477.

Bolger, E. (2002). Characterizations of two power indices for voting games with $\mathrm{r}$ alternatives. Soc Choice Welfare 19:709-721.

Borkotokey, S. (2008). Cooperative games with fuzzy coalitions and fuzzy characteristic functions. Fuzzy Sets Systems 159:138-151.

Casajus, A. (2011). Marginality, differential marginality, and the Banzhaf value. Theory and Decision 71(3):365-372.

Casajus, A. (2012). Amalgamating players, symmetry, and the Banzhaf value. International Journal of Game Theory 41(3):497-515.

Coleman, J.S. (1971). Control of collectivities and the power of a collectivity to act. In Lieberman, B. editor, Social Choice, pages 269-300. Gordon and Breach. New York, USA.

Courtin, S. and Tchantcho, B. (2020). Public good indices for games with several levels of approval. The B.E. Journal of Theoretical Economics, 20(1):1-20.

Dubey, P., Einy E. and Haimanko, O. (2005). Compund voting and the Banzhaf index. Game and Economic Behavior, 51(1):20-30.

Dubey, P. and Shapley L.S. (1979). Mathematical properties of the Banzhaf power index. Mathematics of Operations Research, 4(2):99-131.

Einy, E. (1985). The desirability relation of simple games. Mathematical Social Sciences, 10(2):155-168.

Felsenthal, D.S. and Machover, M. (1997). Ternary voting games. International Journal of Game Theory, 26(3):335-351.

Felsenthal, D.S. and Machover, M. (1998). The Measurament of Voting Power: Theory and practice, problems and paradoxes. Cheltenham: Edward Elgar.

Feltkamp, V. (1995). Alternative axiomatic characterizations of the Shapley and the Banzhaf values. International Journal of Game Theory, 24(2):179-186.

Freixas, J. (2005). Banzhaf measures for games with several levels of approval in the input and output. Annals of Operations Research, 137(1):45-66.

Freixas, J. (2012). Probabilistic power indices for voting rules with abstention. Mathematical Social Sciences, 64(1):89-99.

Freixas, J. (2020). The Banzhaf value for cooperative and simple multichoice games. Group Decision and Negotiation, 29:61-74.

Freixas, J. and Lucchetti, R. (2016). Power in voting rules with abstention: an axiomatization of two components power index. Annals of Operations Research, 244(2):455-474.

Freixas, J. and Zwicker, W.S. (2003). Weighted voting, abstention, and multiple levels of approval. Social Choice and Welfare, 21(3):399-431.

Freixas, J., Tchantcho B. and Tedjeugang N. (2014). Achievable hierarchies in voting games with abstention. European Journal of Operational Research, 236(1):254-260.

Freixas, J., Tchantcho B. and Tedjeugang N. (2014). Voting games with abstention: Linking completeness and weightedness. Decision Support Systems, 57:172-177. 
Gallego, I., Fernández, A., Jiménez-Losada, A., and Ordóñez, M. (2014). A Banzhaf value for games with fuzzy communication structure: Computing the power of the political groups in the European Parliament. Fuzzy Sets and Systems, 255:128-145.

Haller, H. (1994). Collusion properties of values. International Journal of Game Theory, 23: $261-281$.

Holler, M.J. (1982). Forming coalitions and measuring voting power. Political Studies, $30(2): 262-271$

Hsiao, C.R. and Raghavan, T.E.S. (1992). Monotonicity and dummy free property for multi-choice cooperative games. International Journal of Game Theory, 21(1):301-302.

Hsiao, C.R. and Raghavan, T.E.S. (1993). Shapley value for multichoice cooperative games, I. Games and Economic Behavior, 5(2):240-256.

Isbell, J.R. (1958). A class of simple games. Duke Mathematics Journal, 25(3):423-439.

Jana, J. and Roy, S.K. (2019) Dual hesitant fuzzy matrix games: based on new similarity measure, Soft Computing, 23:8873-8886.

Johnston, R.J. (1978). On the measurement of power: Some reactions to Laver. Environment and Planning A, 10(8):907-914.

Kenfack, J.A.M., Tchantcho, B. and Tsague B.P. (2019). On the ordinal equivalence of the Johnston, Banzhaf and Shapley-Shubik power indices for voting games with abstention. International Journal of Game Theory, 48(2):647-671.

Kurz, S. (2020). A note on limit results for the Penrose-Banzhaf index. Theory and Decision, 88:191-203.

Lehrer, E. (1998). An axiomatization of the Banzhaf value. International Journal of Game Theory, 17: 89-99.

Lidner, I. (2008). A special case of Penroses limit theorem when abstention is allowed. Theory and Decision, 64(4):495-518.

Lidner, I. and Machover, M. (2004). L.S. Penrose's limit theorem: proof of some special cases. Mathematical Social Sciences, 47(1):37-49.

Lidner, I. and Owen, G. (2007). Cases where the Penrose limit theorem does not hold. Mathematical Social Sciences, 53(3):232-238.

Meng, F., Zhang, Q. and Chen, X. (2017). Fuzzy multichoice games with fuzzy characteristic functions. Group Decision and Negotiation, 26, 565-595.

Ono R. (2001). Values for Multialternative Games and Multilinear Extensions. In: Holler M.J., Owen G. (eds) Power Indices and Coalition Formation. Springer, Boston, MA.

Owen, G. (1975). Multilinear extensions and the Banzhaf value. Naval Research Logistics Quarterly, 22(4):741-750.

Owen, G. (1978). Characterization of the Banzhaf-Coleman index. SIAM Journal of Applied Mathematics, 35: 315-327.

Parker, C. (2012). The influence relation for ternary voting games. Games and Economic Behavior, 75(2):867-881.

Penrose, L.S. (1946). The elementary statistics of majority voting. Journal of the Royal Statistical Society, 109(1):53-57.

Pongou, R., Tchantcho, B. and Diffo Lambo L. (2011). Political influence in multichoice institutions: cyclicity, anonymity and transitivity. Theory and Decision, 70(2):157-178.

Taylor, A.D.. and Zwicker, W.S. (1999). Simple games: desirability relations, trading, and pseudoweightings. Princeton University Press, New Jersey, USA, 1999.

Tchantcho, B., Diffo Lambo, L., Pongou, R. and Mbama Engoulou, B. (2008). Voters' power in voting games with abstention: Influence relation and ordinal equivalence of power theories. Games and Economic Behavior, 64(1):335-350. 